EPJ manuscript No.

(will be inserted by the editor)

\title{
Inwardly Curved Polymer Brushes : Concave is not Like Convex
}

\author{
M. Manghi ${ }^{1}$, M. Aubouy ${ }^{1}$, C. Gay ${ }^{2}$, and C. Ligoure ${ }^{2,3}$ \\ 1 Groupe Théorie, SI3M, UMR 5819 (CEA-CNRS-Univ. J. Fourier), Département de Recherche Fondamentale sur la Matière \\ Condensée, CEA-Grenoble, 38054 Grenoble cedex 9, France, e-mail: mmanghi@cea.fr, maubouy@cea.fr \\ ${ }^{2}$ Laboratoire CNRS - Elf Atochem (UMR 167), B.P.108, 92303 Levallois-Perret cedex, France, e-mail: cgay@pobox.com \\ ${ }^{3}$ GDPC CC 26, Université Montpellier 2, 34095 Montpellier Cedex 05, France, e-mail: ligoure@gdpc.univ-montp2.fr
}

October 25, 2018

\begin{abstract}
Inwardly curved polymer brushes are present in cylindrical and spherical micelles or in membranes tubes and vesicles decorated with anchored polymers, and influence their stability. We consider such polymer brushes in good solvent and show that previous works, based on a self-similar concentric structure of the brush, do not describe the most stable structure. We use scaling laws to derive very simply the leading term of the free energy in the high curvature limit, where the osmotic pressure is the relevant physical ingredient. We also derive the complete conformation at all curvatures using a self-consistent field approach. The free energy is computed therefrom using a local scaling description
\end{abstract}

PACS. 3 6.20.Ey Conformations (statistics and dynamics) - 82.35.Gh Polymers on surfaces, adhesion 82.70.-y Disperse systems; complex fluids

\section{Introduction.}

Various types of polymer layers have been studied in recent years. The most common situations include polymer ' brushes (where polymers molecules are end-tethered to a repulsive surface or interface), reversible adsorption from a dilute polymer solution, irreversible adsorption from a melt. These are important in the field of adhesion, wet' ting, lubrication, colloid stabilization [1, 2, 3]. Different theories have been proposed to describe the structure and the properties of the polymer coatings as a function of the mode of attachment, the quality of the solvent, etc. However, concerning the geometry of the surface which supports the layer, most of the work is concerned with flat or convex interfaces [4,5]. This is in contrast with a number of situations where polymers chains are attached to concave surfaces. For example, diblock copolymers can form spherical micelles in a selective solvent. The core of these is made of the segregated moeties and can be pictured as a dense layer of chains "grafted" to the inner surface of a sphere. Recently, nine different phases have been found in a ternary system of poly(ethylene oxide)/poly(propylene oxide) amphiphilic block copolymer, water and oil (selective solvents). Six of these phases are made of cylindrical and spherical normal micelles (water-in-oil) or reverse micelles (oil-in-water) [6]. Lattice Monte Carlo simulations were performed to study chain conformations in spherical cavities [7]. Polymers attached to fluid membranes provide another interesting situation. Indeed, in biological systems, lipid bilayers are often decorated by a large number of macromolecules. Inspired by biological structures, simplified model systems of polymers attached to lipid bilayers have been studied experimentally [8,9, 10,11]. It was shown that anchoring polymers induces dramatic changes in the vesicles [8] or multilamellar membrane tubes [11] shapes. In general, the polymers will be attached to both sides, i.e. to both monolayers of the lipid or surfactant bilayer. Anchored polymers also stabilize the equilibrium formation of small unilamellar surfactant vesicles [12 over a wide range of composition and temperature range: the vesicles are spontaneously spherical and monodisperse and their radius is small (comparable to the natural size of the anchored polymers). This spontaneous tendency for bilayers to bend so to form vesicles has been explained by a coupling 13 between the curvature and the asymmetry of grafting polymers on each monolayer 14].

Polymer adsorption finds extensive applications in modifying physical and chemical properties of surfaces. In this context, experiments have highlighted the effect of surface roughness at the size scale of the grafting polymers on the total amount of polymer adsorbed [15]. A significant enhancement of adsorption was observed when the bead to polymer radius is of order of unity, indicating that polymer brushes will prefer to adsorb on spherical or cylindrical bumps (i.e. defects of large positive curvature), since the deformation energy of the brush will be lowered in these geometries with respect to the flat case 16]. On the contrary, valleys, (i.e. defects with large negative curvature) substantially reduce the grafting. Such a reduction was understood in terms of topologically reduced steric hindrance to adsorption. Monte-Carlo computer simulations were also performed [17] to study the adsorption of 
M. Manghi et al.: Inwardly Curved Polymer Brushes : Concave is not Like Convex

triblock copolymers on rough surfaces and confirm that grafting on defects of negative curvature (concave surfaces) is not favoured because of conformational energy penalty of the anchored polymers.

Surprisingly, even if it was anticipated that in the high curvature limit, concave and convex brushes should not be described in a similar way [16], very few theoretical works have been devoted to the specific case of inwardly curved brushes up to date. To our knowledge, only a few theoretical papers have addressed this problem, two of them in the context of the study of the elastic properties of polymerdecorated membranes [18,19], one in the context of shear swelling of polymer brushes grafted onto convex and concave surfaces [20], two in the context of microemulsion stabilization by diblock copolymers [21,22]. E. Zhulina [23] also pointed out references [24,25]. However, most of these authors described the chain conformation and the free energy of the brush on the concave side of the interface by using an inverted version of the classical Daoud-Cotton scaling approach [26]. In this approach, the structure of the brush is self-similar and scales linearly with the distance from the center of curvature (Figure 1). It correctly describes brushes anchored on the convex side of interfaces but not on concave side, because, as we will show below, this structure is not the most stable one.

The main concern of the present work is to provide a correct description of the concave brush structure. In order to clarify the situation of interest, let us briefly review the different conformation regimes (Figure 2), depending on the number $f$ of polymer chains (assumed to be linear, flexible, made of identical, neutral monomers of size $a$ ), on the number $N$ of monomers per chain and on the radius $R$ of the inner surface of the sphere. The number of chains per unit surface area of chains (grafting density) can be written adimensionally as $\sigma=a^{2} f /\left(4 \pi R^{2}\right)$. For simplicity reasons, we only treat the case of an athermal solvent : the excluded volume parameter is $v \cong 1$ (in units of $a^{3}$ ). We assume that the chains are repelled by the interface. An important practical issue requiring attention is the case of flexible membranes with grafted polymers. From this point of view, we will now express all quantities in terms of the grafting density, $\sigma$, rather than in terms of the number of chains, $f$. It allows also an easier comparison with the limit case of a flat brush.

At large sphere radius $R$, if chains are grafted too far apart to interact, they form separate mushrooms (regime 1 of Figure 2) whose Flory radius of gyration is $R_{F}=a N^{3 / 5}$. They start to interact when $\sigma \cong\left(a / R_{F}\right)^{2}$, i.e., when :

$$
\sigma \simeq N^{-6 / 5}
$$

For larger values of the grafting density $\sigma$, chains are stretched in good solvent and form a brush whose height is essentially the same as in the planar geometry :

$$
L_{\text {flat }} \cong a N \sigma^{1 / 3}
$$

This is regime 2 of Figure 2. It is limited by two phenomena. First, the grafting density cannot exceed one chain per squared monomer size, for obvious steric reasons. This condition reads :

$$
\sigma \leq 1
$$

Secondly, the sphere curvature becomes important when $R \simeq L_{\text {flat }}$, i.e., when :

$$
R \simeq a N \sigma^{1 / 3}
$$

At smaller radii, the description of the grafted chain conformation is not straightforward since both the curvature of the sphere and the interaction between neighbouring chains are important. These are regime 3 (where chains are still stretched) and regime 4 of Figure 2 (where chains are confined). The separation of these two regimes occurs when the radius is equal to the natural size of a chain in a semi-dilute solution (see Section 3.2), i.e. when

$$
R \simeq a \sigma^{-1 / 7} N^{3 / 7}
$$

They are limited by two conditions: one occurs when no more solvent is present :

$$
R \geq a N \sigma
$$

and the second one when there is only one chain in the sphere :

$$
R \geq a \sigma^{-1 / 2}
$$

In regimes 2 and 3, chains are stretched and their conformations should play an important role in the structure and the energy of brushes in these regimes. We thus briefly review two usual types of descriptions. In scaling descriptions, all chain conformations are identical and in particular, chain ends are all located at the same distance from the grafting interface. Conversely, self-consistent field (SCF) descriptions allow for a spatial distribution of chain ends. Correlatively, all chain conformations are not identical : at any given distance from the wall, chains are stretched differently according to how far from the grafting surface their free ends are located.

The common feature of both approaches is that the brush conformation results from the interplay between the two-body interactions between monomers pertaining to the brush chains (which tend to stretch the brush further out) and the elastic stretching of the brush chains (which limits the brush extension). As long as these two components are well taken into account, both types of approaches yield essentially similar conclusions.

Thus, the Daoud-Cotton description of convex brushes, although based on scaling arguments, yields correct results. Let us emphasize a few features of such a system. In reality, free chain ends can sustain no stretching, whereas the grafted ends are under tension. In the model by Daoud and Cotton, the typical blob size (see Figure 1a) is larger at greater distances from the wall (it is proportional to the distance from the center of curvature). Thus local stretching is weaker far from the wall. This feature ensures local equilibrium of the chain strands: the gradient in concentration and thus in two-body interactions tends to push them away from the wall, whereas the gradient in degree 
of stretching tends to pull them back towards the wall. If we focus on the last blob, the elastic force, given by the Pincus law 27], is $k_{B} T / \xi(L)$, where $\xi(L)$ is the size of the last blob. Since it is large, the elastic force is small and is balanced by the small concentration gradient at the edge of the brush. The success of this scaling vision is thus due to the fact that chain-ends are segregated in the last blob and they sustain almost no stretching. Hence, the basic ingredients are thus explicitely present in this scaling description.

Convexity of the interface is a key feature in this success of the Daoud-Cotton model : the planar geometry is a marginal case for the Alexander-de Gennes scaling model. It yields essentially the same results (brush extension and free energy) as the pionneering SCF description by Semenov 28] which was further developed independently by two groups [29,30]. However, the monomer concentration profile found in the SCF theory is parabolic and vanishes at the end of the brush. This is different from the step profile of the scaling description which imposes a sharp density gradient at the edge. We might argue that this gradient balances the elastic restoring force exerted on the stretched chain and that it ensures the global equilibrium of chains. But it has been shown that this is not the most stable structure since the SCF theory yields a lower minimum free energy.

Things get worse in the case of a concave geometry. Two scaling descriptions can be found in literature : one, adapted from the model by Daoud and Cotton 18, 19,21,24, the other, given by Sevick [20], is based on an Alexanderde Gennes brush treated locally to account for a cylindrical geometry. The essential feature of the "inverse" DaoudCotton approach is that the blob size is still taken as proportional to the distance from the center of curvature : because the interface is concave, the blob size decreases as we go further away from the wall (Figure 1b). The essential results of this model are as follows (Throughout this article, free energies $F$ are in units of thermal energy $\left.k_{B} T\right)$ :

- the thickness of the brush is $L_{D C} \simeq R\left[1-\left(1-\frac{a N \sigma^{1 / 3}}{R}\right)^{3 / 5}\right]$

- the free energy per chain is $F_{D C} \simeq-N \sigma^{5 / 6} \frac{\ln \left[1-a N \sigma^{1 / 3} / R\right]}{a N \sigma^{1 / 3} / R}$

- the volume fraction profile is $\phi(r) \simeq \frac{\sigma^{2 / 3}}{(1-r / R)^{4 / 3}}$ where

$r$ is the radial distance to the spherical surface (see Figure 1bis)

Among these predictions, the last one is certainly the most surprising. It predicts that the volume fraction increases when we move off from the surface, and notably, at the edge of the brush, we find $\phi(L) \simeq \frac{\sigma^{2 / 3}}{\left(1-a N \sigma^{1 / 3} / R\right)^{4 / 5}}$. Thus forces exerted on the last blob (osmotic gradient and elastic force) are very important. Eventually, the volume fraction diverges at the center for $R \simeq a N \sigma^{1 / 3}$. Hence, even if the local stability inside the brush is fulfilled, as in the convex case, we might expect that this profile does not give the best answer.

Among the results yielded by the Sevick model, one is particularly surprising: at constant grafting density, the brush height is predicted to decrease if the interface is curved more strongly. Yet simple arguments indicate that on the contrary, the brush height should increase. Suppose for a moment that the height does not change when the curvature is changed. Curving the interface more strongly then reduces the overall volume of the brush. Two-body interactions are then more important, which tends to swell the brush. The height will therefore stabilize at a somewhat greater value.

A third approach considers the droplet curvature effect on the emulsion stabilization by diblock copolymers [22]. They study the small corrections due to curvature to the flat case in the framework of the Alexander-de Gennes model, which is valid for very weak curvatures.

To describe such concave brushes under large curvatures, one must therefore keep in mind that the curved brush is some kind of a compressed brush. Curving the interface indeed amounts to increasing the brush concentration : the corresponding compression does not originate in an interaction at the brush edge, however, but rather in some kind of lateral interactions (and the brush profile is very different from that of a compressed planar brush, as we indicate in Appendix A). In this context, one result obtained by Rabin and Alexander on compressed polymer brushes is most important 31]: they showed that, when the layer thickness is forced to be smaller than that of a free brush (which here corresponds to regimes 3 and 4), the main contribution to the free energy of a compressed brush in good solvent is the osmotic contribution. The elastic stretching energy of the chains is comparatively smaller, even though they are still strongly stretched (at least in regime 3 ). As a result, in the limit of strong compressions (regimes 3 and $4, R \ll a N \sigma^{1 / 3}$ ), the osmotic pressure is the mostly contributing term and is therefore uniform over the whole brush volume. In other words, the monomer concentration is uniform over the entire brush and the brush structure is locally that of a semi-dilute solution of equal overall concentration.

The size of the semi-dilute blobs is $\xi \simeq a \phi^{-3 / 4}$ (where $\phi=3 a N \sigma / R$ is the volume fraction) and the free energy of the whole brush is therefore :

$$
F_{3-4}^{\mathrm{sph}} \simeq \frac{R^{3}}{\xi^{3}} \simeq(N \sigma)^{9 / 4} R^{3 / 4}
$$

This expression for the energy is valid well inside regime 3 or in regime 4 , i.e., when the brush is strongly compressed $\left(R \ll L_{\text {flat }}\right)$.

Our aim is to give a correct description of the chain structure and free energy in regimes 2 and 3 . The present work is organized as follows.

We present in Section 2 a SCF approach for such concave brushes valid for a near-theta solvent and an alternative convolution method is outlined in Appendix A. The purpose of Section 3 is to give a scaling picture of concave brushes by refining SCF results taking into account correlations between monomers. A discussion of lateral fluctuations of grafted chains is given in Appendix B, where it is shown that they do not alter significantly the chain free energy and thus do not endanger the SCF approach. 
In Appendix C, we give the results obtained in the cylindrical geometry through a scaling approach.

\section{Self-consistent mean field description.}

In regimes 2 and 3 , the polymer chains are strongly stretched and it is thus possible to use the self-consistent field method [28, 29, 30]. We note $r$ the radial distance to the spherical surface (defined by $r=0$ ).

The aim of this section is to determine the free energy $F$ by solving for the monomer volume fraction $\phi(r)$ and for the distribution $g(r)$ of chain-end monomers selfconsistently. In the classical limit of strong stretching [29], the free energy of the system is

$$
\begin{aligned}
F & =\int_{0}^{R} g\left(r_{0}\right) \int_{0}^{r_{0}}\left[\frac{3}{2 a^{2}} e\left(r, r_{0}\right)+v \frac{\phi(r)}{e\left(r, r_{0}\right)}\right] d r d r_{0} \\
& -\frac{1}{2} \int_{0}^{R} \frac{4 \pi(R-r)^{2} v}{a^{3}} \phi^{2}(r) d r
\end{aligned}
$$

where $g\left(r_{0}\right) d r_{0}$ is the number of chain ends in the spherical shell of radius $R-r_{0}$ and width $d r_{0}$ and $e\left(r, r_{0}\right)=\left|\frac{d r}{d n}\right|$ is the local extension of a chain whose free end is at $r_{0}$ (the variable $n$ denotes the current monomer). The first term corresponds to a sum of single-chain free energies in the potential $k_{B} T v \phi(r)$, the second term corrects the fact that monomer-monomer interactions have been doublecounted. The normalization conditions

$$
\begin{gathered}
\int_{0}^{r_{0}} \frac{d r}{e\left(r, r_{0}\right)}=N \\
\int_{0}^{R} 4 \pi(R-r)^{2} \phi(r) d r=4 \pi R^{2} a N \sigma
\end{gathered}
$$

fix the number of monomers in one chain and in the whole system. The mean field concentration is determined by $\delta F / \delta \phi=0$, which yields

$$
\phi(r)=\frac{a^{3}}{4 \pi(R-r)^{2}} \int_{r}^{R} \frac{g\left(r_{0}\right)}{e\left(r, r_{0}\right)} d r_{0}
$$

We perform this calculation by two steps [29]: first we suppose that the brush height, $x R$, is fixed, and we minimize the free energy given by Eq. (9) with respect to changes in the field $\phi$. This yields a family of profiles $\phi_{\min }$. Then we determine this unknown parameter $x$ (with $0 \leq x \leq 1)$ by minimization of $F\left[\phi_{\min }, x\right]$ which yields the brush height, $x^{*}$, at equilibrium.

The "equal-time" property can be applied for the selfconsistent field as was done previously in the case of a melt 28 if the following two assumptions hold : a) there is no dead zone containing no chain ends [32], which can be written as $g\left(r_{0}\right) \neq 0$ for $0<r_{0} / R<x$; and $b$ ) the chain ends are not under tension : $e\left(r_{0}, r_{0}\right)=0$. From the equaltime argument, we know that the monomer concentration profile is parabolic and we know the form of the local extension of the chain :

$$
\begin{gathered}
\phi_{\min }(r)=\Phi\left[A-B\left(\frac{r}{R}\right)^{2}\right] \Theta(r-x R) \\
B=\frac{\pi^{2} R^{3}}{8 \sigma N^{3} a^{3} v}=\frac{10}{9}\left(\frac{R}{R^{*}}\right)^{3}
\end{gathered}
$$

where

$$
\begin{gathered}
R^{*}=\left(\frac{80}{9 \pi^{2}}\right)^{1 / 3} a(v \sigma)^{1 / 3} N \\
e\left(r, r_{0}\right)=\frac{\pi}{2 N} \sqrt{r_{0}^{2}-r^{2}}
\end{gathered}
$$

where $\Theta$ is a step function, $\Phi=3 a N \sigma / R, B$ is obtained from the equal-time argument (Eq. (10)) and $A$ is related to $x$ by (Eq. (11))

$$
\left(3-3 x+x^{2}\right) x A=1+\frac{10}{9}\left(\frac{R}{R^{*}}\right)^{3} x^{3}\left(1-\frac{3}{2} x+\frac{3}{5} x^{2}\right)
$$

The minimization of the free energy per chain $\widetilde{F}(x)=$ $F\left[\phi_{\min }, x\right] / f$ with respect to changes in $x$ under the three conditions a) $0 \leq x \leq 1$, b) $\phi\left(x^{-}\right) \geq 0$ and c) $\phi(0)=$ $\Phi A \leq 1$ is illustrated in Figure 3. This gives rise to a distinction between two cases depending on whether $R$ is smaller or larger than $R^{*}$, where $R^{*}$ (see Eq. (14)) is the critical radius for which the sphere is entirely filled up by the brush:

- for $R>R^{*}$ (regime 2), the minimum free energy $\widetilde{F}$ occurs for $x^{*}$ defined has the unique solution of the equation

$$
x^{3}\left(\frac{4}{9} x^{2}-\frac{5}{3} x+\frac{20}{9}\right)=\left(\frac{R^{*}}{R}\right)^{3}
$$

such as condition $a$ ) is fulfilled (Note that this condition is equivalent to the smooth vanishing of the polymer concentration at the edge of the brush [34]). It remains a central region of radius $R\left(1-x^{*}\right)$ in the center of the sphere which contains pure solvent ;

- for $R \leq R^{*}$ (regime 3 ) the minimum of $\widetilde{F}$ occurs for $x=1$, there is no empty region and the concentration at the center of the sphere is finite.

By varying $R$ from $\infty$ to $a N^{1 / 2}$, the limit of validity of the SCF approach, the following scenario emerges.

\subsection{Regime $2\left(R>R^{*}\right)$}

In regime 2 , the brush height $x^{*}$ is given by Eq. (18) and the monomer volume fraction (Eq. (13)) simplifies into [35] (Figure 4)

$$
\phi(r)=\Phi B\left[x^{* 2}-\left(\frac{r}{R}\right)^{2}\right]
$$


Equations (12) and (19) yield the chain end distribution written in units of chains per unit length $g(t)=\frac{g\left(r_{0}\right) a^{2}}{4 \pi R \sigma}$ :

$$
\begin{aligned}
g(t) & =B x^{* 2} t\left[3\left(2-3 x^{*}+2 x^{* 2}\right) \sqrt{1-t^{2}}-8 x^{* 2}\left(1-t^{2}\right)^{3 / 2}\right. \\
& \left.+6 x^{*} \arg \tanh \sqrt{1-t^{2}}-9 x^{*} t^{2} \arg \tanh \sqrt{1-t^{2}}\right]
\end{aligned}
$$

where $t=r_{0} /\left(x^{*} R\right)$. The distribution of chain ends can also be expressed in terms of a number of chain ends per unit volume $: \rho(t)=\frac{g(t)}{3\left(1-t x^{*}\right)^{2}}$ (in units of chains per unit volume, $\Phi / N)$ for $0<t<1$. A typical probability density for free chain ends is shown in Figure 5.

¿From these, we readily derive the free energy per chain $\widetilde{F}_{2}=\widetilde{F}\left(x^{*}\right)$ given by Eq. (9) :

$$
\widetilde{F}_{2}=\frac{9}{10}\left(\frac{\pi^{2}}{4}\right)^{1 / 3} N(v \sigma)^{2 / 3} \psi\left(\frac{R^{*}}{R}\right)
$$

where

$$
\begin{aligned}
\psi(y) & =\frac{10 \cdot 4^{1 / 3}}{3\left(3-3 x^{*}+x^{* 2}\right)}\left[\frac{(9 / 10)^{1 / 3}}{4} \frac{y}{x^{*}}\right. \\
& +\frac{(25 / 6)^{1 / 3}}{3} \frac{x^{* 2}}{y^{2}}\left(1-\frac{3}{2} x^{*}+\frac{3}{5} x^{* 2}\right) \\
& \left.-\frac{(25 / 6)^{1 / 3}}{189} \frac{x^{* 5}}{y^{5}}\left(28-63 x^{*}+\frac{201}{4} x^{* 2}-17 x^{* 3}+\frac{12}{5} x^{* 4}\right)\right]
\end{aligned}
$$

In the entire regime 2 , equation (18) shows that the normalized brush height $x^{*}$ increases when the curvature $R^{*} / R$ increases, until it reaches $x^{*}=1$ for $R=R^{*}$ (Figure 6). In other words, the brush height increases when the interface is curved more strongly, as long as there is some free solvent in the center. In the same way, it can be shown that the normalized free energy $\psi$ (Eq. (23)) increases monotonically at constant grafting density when the curvature increases (Figure 7).

The osmotic pressure balances the elastic restoring force since the polymer brush is at equilibrium under no external compression. This readily explains [29] the fact that the monomer volume fraction vanishes smoothly at the outer edge of the brush (equation (18) is equivalent to $\left.\phi\left(x^{*}\right)=0\right)$.

Izzo and Marques [36] seem to have also used an SCF approach for swollen concave brushes in this regime. They find the same equation as Eq.(18) for the brush height, but their free energy is different from the one given here. We do not understand why at this stage.

In the limit of zero curvature, i.e. $R \rightarrow \infty$, the brush height $L=x^{*} R$ given by Eq. 18) can be expressed as :

$$
L_{\text {flat }}=\left(\frac{9}{20}\right)^{1 / 3} R^{*}=\left(\frac{4}{\pi^{2}}\right)^{1 / 3} a(v \sigma)^{1 / 3} N
$$

which is the result of Milner, Witten and Cates 229]. Moreover, we have $\psi(0)=1$ and the energy Eq. (21) becomes

$$
\widetilde{F}_{2 \text { flat }}=\frac{9}{10}\left(\frac{\pi^{2}}{4}\right)^{1 / 3} N(v \sigma)^{2 / 3}
$$

which is also the classical result.

Going further, we can expand this result for very small curvatures. Equation (18) yields the asymptotic value of the brush height

$$
L \simeq L_{\text {flat }}\left[1+\frac{1}{4} \frac{L_{\text {flat }}}{R}+\frac{29}{240}\left(\frac{L_{\text {flat }}}{R}\right)^{2}\right]
$$

Note that a simple scaling method does not yield the correct value for this asymptotic increase in the brush height [37]. We can also expand the free energy for small curvatures

$$
\widetilde{F}_{2}=\widetilde{F}_{2 \text { flat }}\left[1+\frac{5}{12} \frac{L_{\text {flat }}}{R}+\frac{73}{336}\left(\frac{L_{\text {flat }}}{R}\right)^{2}\right]
$$

We observe that this is consistent with the results of Milner and Witten [38] who found for the convex geometry the same development by replacing $R$ by $-R$. This is because, as explained in [38], in the convex case, the dead (22) ne yields negligible terms for small curvatures [39].

In conclusion, for this region 2 , the $\mathrm{SCF}$ calculations show a weak increase of the brush height when the interface is curved more and more, which involves an increase of both elastic and osmotic energy.

\subsection{Regime $3\left(a N^{1 / 2} \leq R \leq R^{*}\right)$}

In regime 3 , the brush height $L$ is fixed at $R(x=1)$. The monomer volume fraction becomes [35] (Figure 4)

$$
\phi(r)=\phi(R)+\Phi B\left[1-\left(\frac{r}{R}\right)^{2}\right]
$$

where $\Phi(R)$ is the volume fraction at the center of the sphere given by :

$$
\phi(R)=\Phi\left[1-\left(\frac{R}{R^{*}}\right)^{3}\right]
$$

which increases when $R$ decreases. Thus, the monomer volume fraction tends to become more uniform as the radius $R$ is decreased.

The chain end distribution is $(t=r / R)$

$$
\begin{aligned}
g(t)= & t\left[-6\left(1-\frac{9}{10} B\right) \sqrt{1-t^{2}}-8 B\left(1-t^{2}\right)^{3 / 2}\right. \\
& \left.+6\left(1+\frac{1}{10} B\right) \arg \tanh \sqrt{1-t^{2}}-9 B t^{2} \arg \tanh \sqrt{1-t^{2}}\right]
\end{aligned}
$$

As is shown in Figure 5, the major number of chain ends per unit surface are located at the edge of the brush. The free energy per chain is given by :

$\widetilde{F}_{3}=\left(\frac{9 \pi^{2}}{10}\right)^{1 / 3}(v \sigma)^{2 / 3} N\left[\frac{3}{4} \frac{R^{*}}{R}+\frac{1}{6}\left(\frac{R}{R^{*}}\right)^{2}-\frac{13}{756}\left(\frac{R}{R^{*}}\right)^{5}\right]$

Mathematically, this free energy $\widetilde{F}_{3}$ is frozen at $\widetilde{F}(x=1)$ because the equilibrium state $x=x^{*}>1$ is out of reach, 
$x=1$ being a physical bound of the problem. This situation is similar to the case of flat brush under compression (a pressure is exerted at its edge): the concentration profile has a truncated parabolic shape and its value at the edge of the brush, Eq. (28), increases when the compression is stronger [40]. As it has been shown by Milner et $a l$., this means that there can be no "interdigitation" of chains at $r=R$ 34].

At constant grafting density, $\widetilde{F}_{3}$ (Eq. (30)) is a strongly increasing function of $R^{*} / R$ (Figure 7). A careful analysis of this energy shows that for $R \ll R^{*}$, the last term is negligible, it comes from the parabolic nature of the monomer concentration. In the same way, from Eq. (28) we can reasonably consider that the monomer concentration is constant and equal to $\Phi=3 N a \sigma / R$. Therefore, the free energy can be seen at the level of a simple Flory theory

$$
\widetilde{F}_{3} \simeq v \frac{a N^{2} \sigma}{R}+\frac{R^{2}}{N a^{2}}
$$

where the first term is the osmotic pressure (at a meanfield level) and the second one the elasticity of an ideal chain. For $R \cong R^{*}$, these two energies balance, but when $R$ decreases further, the excluded volume interactions strongly dominate and the energy is practically equal to the osmotic pressure (Figure 7). The point is that these two energies do not balance because the brush height is geometrically constrained.

\section{Scaling refinements}

In the previous section, we used a self-consistent field approach to study the conformation of a concave brush. Such an approach describes both the average distribution of free chain ends and the average position of any monomer, conditionally if the position of the end-monomer of the chain to which it pertains is known. It is known classically in two versions. The mean-field version such as that exposed in the previous section provides a somewhat erroneous expression for the brush free energy, which varies like $\phi^{2}$. This flaw is intrinsic to the mean-field approximation and is present also in the Flory approach. A correct expression for the free energy can be obtained using scaling concepts [41] and varies like $\phi^{9 / 4}$. It was first used by de Gennes in a scaling SCF approach for the problem of polymer adsorption [42]. Milner, Witten and Cates applied it in order to work out the conformation of a flat, swollen polymer brush in good solvent [34]. The resulting brush concentration profile obtained after minimization is slightly different from that obtained through the mean-field SCF calculation. This is in contrast with the original Alexander-de Gennes model for the brush where both the mean-field and the scaling forms of the free energy yield the very same brush conformation, whose concentration profile is uniform. In the SCF approach, the non-uniformity of the concentration is the origin for the slight difference between mean-field and scaling [43]. In this section, we present these scaling tools for the various regimes of the spherical concave brush. For stretched chains (regimes 2 and 3), we simply calculate the scaling free energy from the brush conformation determined from the mean-field SCF approach exposed in the previous section. Although it is not as rigorous as the calculation of reference [34], it should be a sufficient approximation at least in regime 3 where the concentration profile is essentially uniform, as we just mentioned. In any case, the value of the free energy obtained in this way in regimes 2 and 3 will be improved as compared to the mean-field value obtained in the previous section.

We also describe more fully chain fluctuations around their average conformation given by the SCF treatment. Confined chains (regime 4) cannot be treated by the SCF theory : we describe the chain conformations and calculate the scaling free energy.

\subsection{Scaling refinements in regimes 2 and 3}

The scaling form of the free energy given in reference [34] is :

$$
\begin{aligned}
\widetilde{F}^{s c} & \simeq \frac{1}{2} \int_{0}^{R} \frac{4 \pi(R-r)^{2}}{a^{3}} \phi^{9 / 4}(r) d r \\
& +\frac{3}{2 a^{2}} \int_{0}^{R} d r_{0} g\left(r_{0}\right) \int_{0}^{r_{0}} e\left(r, r_{0}\right) \phi^{1 / 4}(r) d r
\end{aligned}
$$

where the factor $\phi^{9 / 4}$ in the osmotic term comes from the $k_{B} T$ per blob Ansatz, while the $\phi^{1 / 4}$ factor in the elastic term originates in the correction to the unperturbed conformation of the chains which are swollen below the length scale $\xi(r)=a \phi^{-3 / 4}$. The authors interpreted the elastic term as a perturbation of each blob of size $\xi$ : it corresponds to $k T(\Delta \xi / \xi)^{2}$ per blob, where $\Delta \xi$ is the average blob elongation. But this term can be interpreted in a more intuitive way, using a more precise description of the local chain conformation. As mentioned briefly by the authors, at small length scales, chains are like in a semi-dilute solution (swollen below $\xi$ and Gaussian above $\xi$ ), while they are stretched at large length scales. In other words, the root mean square distance between two monomers $n$ and $n^{\prime}$ along the chain (see Figure 8a) is given by :

$$
\begin{array}{rlrl}
\sqrt{\overline{\left(r-r^{\prime}\right)^{2}}} & \propto\left(n-n^{\prime}\right)^{3 / 5} & \left(\sqrt{\overline{\left(r-r^{\prime}\right)^{2}}}<\xi=a \phi^{-3 / 4}\right) \\
& \propto\left(n-n^{\prime}\right)^{1 / 2} \quad \quad\left(\xi<\sqrt{\overline{\left(r-r^{\prime}\right)^{2}}}<\Lambda\right) \\
& \propto n-n^{\prime} \quad\left(\sqrt{\overline{\left(r-r^{\prime}\right)^{2}}}>\Lambda\right)
\end{array}
$$

where the intermediate length scale $\Lambda$, where this transition occurs, is called the elastic blob (see references 44,45]) :

$$
\Lambda\left(r, r_{0}\right) \simeq a^{2}\left|\frac{d r}{d n}\right|^{-1} \phi^{-1 / 4}
$$

At this length scale, the polymer chain is not an ideal random walk any more : instead, it has no choice but to move forward at every step (exponent 1). Hence, the chain is mainly a linear string of these elastic blobs stretched 
along the radial direction; the important point being that these blobs are defined along one direction and that in the other two directions, blobs of different chains overlap.

In the elastic term of equation (9), the degree of stretching $e\left(r, r_{0}\right)$ must be replaced by an effective degree of stretching $e_{\mathrm{eff}}\left(r, r_{0}\right)=a^{2} / \Lambda\left(r, r_{0}\right)$, which yields the factor $\phi^{1 / 4}$ in equation (32).

Note that the osmotic blob size $\xi(r)$ depends only on the position $r$, whereas the size of an elastic blob $\Lambda\left(r, r_{0}\right)$ on a particular chain also depends on the local degree of stretching of that chain, i.e., on the position $r_{0}$ of its free end.

In principle, the chain conformations should be obtained through minimization of equation (32), as in reference 34. Here, however, we simply use the volume fraction $\phi(r)$ (equation (19) or (27)), the free end distribution $g(t)$ (equation (20) or 29) ), and the degree of stretching derived through the mean-field method developed in the previous section (Eq. 16).

The free energy of the brush in regimes 2 and 3 can be computed numerically from equation (32) along the lines we have indicated.

Here, for simplicity, we focus on the limit where $R \ll$ $R^{*}$ (well in regime 3 ). The free end distribution given by equation (29) at zero order in $R / R^{*}$ then becomes :

$$
g(t) \simeq 6 t\left\{\arg \tanh \sqrt{1-t^{2}}-\sqrt{1-t^{2}}\right\}
$$

In the same way, at this order, $\phi(r)$ is constant where $\phi(r)=\Phi=3 a N \sigma / R$. With these two approximations, the total free energy is equal to the first two terms of Eq. (30) multiplied by $\phi^{1 / 4}$ :

$$
\begin{aligned}
\widetilde{F} & \simeq \frac{3}{2} \frac{a N^{2} v \sigma}{R} \phi^{1 / 4}+\frac{3 \pi^{2}}{80} \frac{R^{2}}{N a^{2}} \phi^{1 / 4} \\
& \simeq\left(\frac{3}{2}\right)^{1 / 4}\left(\frac{9 \pi^{2}}{10}\right)^{5 / 12} \sigma^{5 / 6} N\left[\frac{3}{4}\left(\frac{R^{*}}{R}\right)^{5 / 4}+\frac{1}{6}\left(\frac{R}{R^{*}}\right)^{7 / 4}\right.
\end{aligned}
$$

where the dominant contribution is osmotic (first term). The second term is the elastic term (computed above for $\left.R / R^{*} \rightarrow 0\right)$. The next nonzero contributions from the osmotic and the elastic terms in the $R / R^{*}$ (or $B$ ) expansion are smaller by a factor of order $\left(R / R^{*}\right)^{3}$.

Finally, in order to get simply the scaling dependence of respectively the osmotic and elastic energy in regimes 2 and 3 (within unknown numerical coefficients of order unity), it is possible to keep only the scaling dependence of blobs $: \xi \simeq a \Phi^{-3 / 4}$ and $\Lambda \simeq N \Phi^{-1 / 4} / R$ and to assign the energy $k_{B} T$ per blob. Thus, in regime 2 , we recover the very simple estimate of the balance achieved by the stretched chains first presented by Alexander 46. and de Gennes [47]. The compressed brush in regime 3 is then analogous to a flat brush in presence of a semi-dilute solution of monomer volume fraction $\Phi_{b}=\Phi$ (see Figure 2). The parameter $R$ is related to a virtual external osmotic pressure $\Pi \sim \Phi_{b}^{9 / 4}$ which compress the grafted layer 44,47.

\subsection{Confined chains (regime 4)}

The natural size of an $N$-monomer chain in a semi-dilute solution of volume fraction $\phi$ is known to be $R(\phi) \simeq$ $a N^{1 / 2} \phi^{-1 / 8}=\xi(N / g)^{1 / 2}$ where $\xi=a g^{3 / 5}=a \phi^{-3 / 4}$ is the osmotic blob size defined earlier in this section. In regimes 2 and 3, when the sphere radius is decreased, the chains remain stretched as long as their end-to-end extension is larger than the elastic blob size $\Lambda$. This breaks down when the radius of the sphere reaches the natural size of the chain, i.e., $R=R(\phi)$ or :

$$
R \cong a \sigma^{-1 / 7} N^{3 / 7}
$$

For smaller sphere radii (which corresponds to regime 4, see Figure 3), the grafted chains are confined. More precisely (see Figure 8b), the chains are swollen at small length scales, Gaussian at length scales between $\xi$ and $R$, and confined (zero exponent) for larger numbers of monomers 48]. The transition between the Gaussian and the confined statistics corresponds to $G=\phi^{1 / 4} R^{2} / a^{2}$ monomers. At scales larger than $G$ monomers, because of the spherical boundary, the chain has no choice but to stay in the same region of space instead of performing a random walk : globally, it is a string of $N / G$ overlapping blobs of size $R$. Using the same argument as earlier in this section, we conclude that the compression contribution to the overall energy can be estimated as :

$$
\widetilde{F}_{4 e l} \simeq \frac{N}{G} \simeq N^{3 / 4} \sigma^{-1 / 4}(R / a)^{-7 / 4}
$$

This, however, is only a correction to the free energy, whose main contribution is osmotic and is given by equation (8).

\section{${ }^{7 / 4} 4$ Concluding Remarks}

-In this article, we have given both an SCF and a scaling study of the structure and the associated free energy of polymer brushes in concave geometries. A simple scaling description can be derived from SCF calculations. The essential features are the following : for weak curvatures $\left(R>R^{*} \simeq 1.3 L_{\text {flat }}\right.$, where $L_{\text {flat }}$ is the thickness of a planar brush with the same grafting density), inwardly curved brushes are a mere generalization of flat brushes : the monomer concentration decreases as we move off from the surface. A specific scaling description which would account for the geometry and for this slowly decreasing concentration would yield nothing else but constant blobs as in the Alexander-de Gennes description of flat brushes. This relatively poor scaling description can be related to the fact that the chain end distribution spreads over the entire layer. However, we argue that a self-similar profile of inverted Daoud-Cotton type is not the correct scaling description. Even if the variation of the thickness with $R$ does not allow to bring to a firm conclusion, this approach does not lead to the lowest free energy since not only it overestimates the actual free energy but also it yields 
an increasing monomer concentration which diverges in the center for $R \simeq L_{\text {flat }}$. At large curvatures (keeping the grafting density constant), the monomer concentration becomes progressively uniform, leading to the structure of a squeezed semi-dilute solution where monomermonomer interactions predominate over chain stretching energy. This description is consistent with the fact that most chain ends are located at the center of the sphere, just like in a compressed brush where they are located at the edge of the brush. Finally, we also give elements to compute numerically the free energy taking into account correlations between monomers, by using the SCF results for the volume fraction and the chain end distribution. We hope that the diagram of the different regimes according to the values of $R$ and the grafting density $\sigma$ will be checked experimentally in microemulsions [49].

As soon as $R \simeq L_{\text {flat }}$, the dissymmetry between convex and concave cases, which originates in the difference of available space for the brush, becomes important and plays a non negligible role in the stability of micelles and polymer decorated membranes. This article brings the essential elements for the determination of membrane spontaneous curvature, mean and Gaussian rigidity moduli especially for decorated vesicles of high curvatures at thermodynamic equilibrium (where the radius is of order of $100 \AA$ [12]). This theoretical work should also illuminate the issue of the adsorption of polymers on rough surfaces consisting of bumps and hollows of high curvatures [16. However, the question of membrane fluctuations with a short wavelength $\lambda<L_{\text {flat }}$ still awaits consideration since the range of steric interactions between chains is then longer than $\lambda$. The distribution and conformation of grafted polymers should then be altered only in the vicinity of the grafting points. Indications of this have been given experimentally by Singh et al. : the influence of surface roughness on polymer adsorption is smaller when the roughness has a very small wavelength 15 .
The authors wish to thank Alice Nicolas and Bertrand Fourcade for useful discussions. We also thank E. Zhulina for pointing out references [24,25] to us and for raising the argument of the stabilizing concentration gradient at the edge of the brush.

\section{Appendix A: Convolution method for a self- consistent field treatment of the concave brush}

We use here an alternate (and faster) method to calculate the free energy in the SCF theory. As an example, we treat the regime 3 .

We know from the works of Semenov and MWC that the potential profile is parabolic, and that it is proportional to the volume fraction:

$$
\phi(r)=\Phi+\frac{1}{10} C-C \frac{r^{2}}{R^{2}}
$$

where $\Phi=3 a N \sigma / R$ is the average volume fraction is calculated by normalization and where $C=3 \pi^{2} R^{2} / 8 a^{2} v N^{2}$ is found from the "equal-time argument". In regime 3 , the volume fraction has some finite value both at the center $\phi(R) \equiv \Phi-9 / 10 C$. Taking advantage of these results, we use the convolution method outlined in reference [50] to determine the free energy (in mean-field). From this we calculate the two-body excluded volume interaction free energy of the brush :

$$
\begin{aligned}
F_{\text {os }} & =\frac{1}{2} \int 4 \pi(R-r)^{2} d r \frac{v}{a^{3}} \phi^{2}(r) \\
& =\frac{2 \pi R^{3} v}{a^{3}}\left\{\frac{1}{3} \Phi^{2}+\frac{13}{2100} C^{2}\right\}
\end{aligned}
$$

Defining now $\phi_{\text {flat }}(r)$ by

$$
4 \pi R^{2} \phi_{\text {flat }}(r) \equiv 4 \pi(R-r)^{2} \phi(r)
$$

as the density profile obtained by flattening out the concave brush like a chopped pineapple slice, we compute the elastic energy [50]:

$$
\begin{aligned}
F_{e l} & =4 \pi R^{2} \frac{\pi^{2}}{8 N^{2} a^{5}} \int_{0}^{R} d r\left\{-r^{3} \frac{d \phi_{\text {flat }}(r)}{d r}\right\} \\
& =\frac{\pi^{3} R^{5}}{2 N^{2} a^{5}}\left\{\frac{1}{10} \Phi-\frac{13}{700} C\right\}
\end{aligned}
$$

Gathering all these results, we find the free energy in the mean field approach (equation (30)) :

$$
F=\frac{2 \pi}{3} \frac{R^{3} v}{a^{3}} \Phi^{2}+\frac{\pi^{3}}{20} \frac{R^{5}}{a^{5} N^{2}} \Phi-\frac{39 \pi^{5}}{22400} \frac{R^{7}}{a^{7} v N^{4}}
$$

The free-end density profile is then obtained by a simple convolution with the volume fraction profile [50] :

$$
\frac{g\left(r_{0}\right)}{4 \pi(R-r)^{2}}=\int_{r_{0}}^{R}\left\{-\frac{d \phi_{\text {flat }}}{d r}(r)\right\} \frac{r_{0}}{N a \sqrt{r^{2}-r_{0}^{2}}} d r(44)
$$

where the last factor is the MWC free end distribution for a melt brush (step-like volume fraction profile). Through integration, we recover equation (29). 


\section{Appendix B: Do lateral fluctuations ruin the SCF approach ?}

The SCF approach describes only a projection of the chain conformation along the normal to the surface and ignores the chain fluctuations parallel to the surface. For a brush grafted to a flat interface, the lateral fluctuations can be described in very simple terms since the chain performs a random walk in these directions : the square-averaged position of the $n$-th monomer from the wall is $y \equiv a n^{1 / 2}$ in the case of a melt, and more generally $y \equiv a n^{1 / 2} \phi^{-1 / 8}$ if $\phi$ is the monomer volume fraction.

Such lateral fluctuations are not hindered in any way for a flat brush since the potential is constant in the lateral directions. In the case of a concave brush, however, when a chain fluctuates laterally, it explores regions located closer to the grafting, concave surface, and the potential is somewhat higher there. In regions where this potential increase becomes of order $k_{B} T$, we expect a reduction in the lateral fluctuations, which in turn represents an additional reduction of entropy. In principle, the potential should therefore be altered accordingly. We now estimate this effect for the regime 3 (where it should be more important) using a scaling description of the chain conformations.

Consider a point at a distance $r$ from the wall where the potential has a certain value $V(r)$. Let us now consider the potential as a function of the distance $z$ from the first point, going perpendicularly to the radius : from geometry, the new point is closer to the wall by $\Delta r \simeq z^{2} / 2(R-r)$. As a consequence, the potential is increased by $\Delta r|d V / d r|$. By considering correlations between monomers, the potential equation (14) is modified according to [34]: $V\left(r^{\prime}\right) / k_{B} T=$ $\phi^{5 / 4}\left(r^{\prime}\right)=\phi^{5 / 4}\left(r^{\prime}(R)\right)+3 \pi^{2} /\left(8 N^{2}\right)\left(r^{\prime 2}(R)-r^{\prime 2}\right)$ where $d r^{\prime}=\phi^{1 / 8}(r) d r$ and $\phi\left(r^{\prime}\right) \equiv \phi(r)$. Hence, the variation of the potential in the perpendicular direction is given by $\left(d V / d r^{\prime}\right)\left(d r^{\prime} / d r\right) \Delta r$.

In regime 3 , the volume fraction is essentially uniform and we have $r^{\prime} \simeq r \phi^{1 / 8}$ thus

$$
V(r, z) \simeq V(r)+k_{B} T \phi^{1 / 4} \frac{z^{2} r}{N^{2}(R-r)}
$$

In the direction of $z$, the chain evolves in the potential given by the above equation. At small length scales, the potential increase is weak and does not affect the chain conformation. At large length scales, the chain is confined in this potential. At the crossover between these two regimes, the typical distance $z$ that is actually explored by the chain is such that the monomers required for a free random walk over that distance, each of which being at a potential of order $V(r, z)$, correspond to an energy of order $k_{B} T$ altogether. In other words, at a given radial position $r$, the lateral fluctuations of the chain may not exceed $z$ given by :

$k_{B} T \simeq \frac{\bar{z}^{2} \phi^{1 / 4}}{a^{2}}[V(r, \bar{z})-V(r)]$ i.e. $\bar{z} \simeq a N^{1 / 2} \phi^{-1 / 8}\left[\frac{R-r}{r}\right]^{1 / 4}$

As $\phi$ is essentially uniform, the lateral fluctuations depend on position only through the last factor $[(R-r) / r]^{1 / 4}$.
Near the wall, the spontaneous fluctuations are given by :

$$
z \simeq a n^{1 / 2} \phi^{-1 / 8} \quad(r \ll R)
$$

since this is still lower than $\bar{z}$. By contrast, close to the center, the lateral fluctuations are given by :

$$
z \simeq a N^{1 / 2} \phi^{-1 / 8}[1-r / R]^{1 / 4} \quad(R-r \ll R)
$$

The energy involved in this effective chain confinement is $k_{B} T$ per blob of size $z$, i.e., per $G_{z} \simeq(z / a)^{2} \phi^{1 / 4} \simeq$ $N[(R-r) / R]^{1 / 2}$ monomers. Now, from the SCF chain conformation $r=R \sin [\pi n / 2 N]$ (if the free end is at the center $r=R$ ), we deduce that $(R-r) / R \sim(N-n)^{2} / N^{2}$. Hence, the free energy due to the restriction of the lateral fluctuations is given by :

$$
F_{\mathrm{conf}} \simeq \int_{0}^{O(N)} \frac{d(N-n)}{G_{z}} \simeq \ln N
$$

As a consequence, the free energy that corresponds to the reduction of the lateral fluctuations is negligible. The fluctuations should be included, however, to fully describe the chain conformations near the center (for $R-r \ll R$ ).

\section{Appendix C: Cylindrical geometry.}

Following the same approach as in the Introduction, a scaling diagram can be established for concave cylindrical brushes in terms of the cylinder radius $R$ and the grafting density $\sigma$ (Figure 9). It should be noticed that two new regimes are present, as compared to the spherical geometry :

- regime 1bis where the cylinder is so small $\left(R<a N^{3 / 5}\right)$ that mushrooms are confined by the cylinder and stretched along the cylinder. This ensures the blob size equal to $R$ and their length is $R_{\|} \simeq N / R^{2 / 3} 41$.

$1 / 4$ regime 4 which is semi-dilute and where chains are not laterally compressed. Their length is $R_{\|} \simeq a N^{1 / 2} \phi^{-1 / 8}$.

The limit between these two regimes is $R \simeq 1 / N^{3} \sigma^{3}$. 


\section{References}

1. D. Napper, Polymeric Stabilisation of Colloidal Dispersions (Academic Press, London, 1983).

2. G. J. Fleer, M. A. Cohen Stuart, J. M. H. M. Scheutjens, T. Cosgrove, B. Vincent, Polymer at Interfaces (Chapman et Hall, London, 1993).

3. P.-G. de Gennes, Advances in Colloid and Interface Science 27, 189 (1987).

4. G. A. McConnell, E. K. Lin, A. P. Gast, J. S. Huang, M. Y. Lin, S. D. Smith, Faraday Discuss. 98, 121 (1994), and references therein.

5. A. Halperin, M. Tirrell, T. P. Lodge, Adv. Polym. Sci. 100, 1 (1991).

6. P. Alexandridis, U. Olsson, B. Lindman, Langmuir 14, 2627 (1998).

7. K. Prochazka, J. Phys. Chem. 99, 14108 (1995).

8. G. Decher, E. Kuchinka, H. Ringsdorf, J. Venzmer, D. Bitter-Suermann, C. Weisgerber, Angew. Makromol. Chem. 166/167, 71 (1989).

9. J. Simon, M. Kühner, H. Ringsdorf, E. Sackman, Chem. Phys. Lipids 76, 241 (1995).

10. G. Blume, G. Cevc, Biochim. Biophys. Acta 1029, 91 (1990).

11. I. Tsafrir, D. Sagi, T. Arzi, M.-A. Guedeau-Boudeville, V. Frette, D. Kandel, J. Stavans, Phys. Rev. Lett. 86, 1138 (2001).

12. R. Joannic, L. Auvray, D. Lasic, Phys. Rev. Lett. 78, 3402 (1997).

13. S. A. Safran, P. A. Pincus, D. Andelman, F. C. MacIntosh, Phys. Rev. A. 43, 1071 (1991).

14. G. Porte, C. Ligoure, J. Chem. Phys. 102, 4290 (1995).

15. N. Singh, A. Karim, F. S. Bates, M. Tirrell, K. Furusawa, Macromolecules 27, 2586 (1994).

16. C. Ligoure, L. Leibler, Macromolecules 23, 5044 (1990).

17. A. C. Balazs, K. Huang, C. W. Lantman, Macromolecules 23, 4641 (1990).

18. C. Hiergeist, R. Lipowsky, J. Phys. II France 6, 1465 (1996).

19. K. Hristova, D. Needham, J. Colloid Interf. Sc. 168, 302 (1994).

20. E. M. Sevick, Macromolecules 29, 6952 (1996).

21. N. Dan, M. Tirrell, Macromolecules 26, 637 (1993).

22. E. N. Govorun, I. Erukhimovich, Langmuir 15, 8392 (1999).

23. E. B. Zhulina (private communication).

24. E. B. Zhulina, Vysokomol. Soedin. B25, 834 (1983).

25. Y. V. Liatskaya, T. M. Birshtein, E. B. Zhulina (unpublished).

26. M. Daoud, J.-P. Cotton, J. Phys. 43, 531 (1982).

27. P. Pincus, Macromolecules 9, 386 (1976).

28. A. N. Semenov, Sov. Phys. JETP 61 (4), 733 (1985).

29. S. T. Milner, T. A. Witten, M. E. Cates, Europhys. Lett. 5, 413 (1988).

30. A. M. Skvortsov, A. A. Gorbunov, V. A. Pavlushkov, E. B. Zhulina, O. V. Borisov, V. A. Priamitsyn, Polym. Sci. USSR 30, 1706 (1988).

31. Y. Rabin, S. Alexander, Europhys. Lett. 13 (1), 49 (1990).

32. The fact that there is no dead zone for the extremities is crucial for applying this SCF method to concave brushes. A dead zone is present for a convex geometry 28.

33. Mathematically, condition $b), \phi\left(x^{-}\right) \geq 0$ is equivalent to $x \leq x^{*}$.
34. S. T. Milner, T. A. Witten, M. E. Cates, Macromolecules 21, 2610 (1988).

35. In principle, the condition $c$ ) is not fulfilled for every value of parameters $(\sigma, R)$. In region 2 , the condition $c$ ) is rewritten $L \leq(2 \sqrt{2} / \pi \sqrt{3}) v^{1 / 2} N$ which is valid for every radii $R$ if $\sigma \leq \sigma_{1}=(\sqrt{6} / 5 \pi) v^{1 / 2}$. In region 3 , if $\sigma \leq \sigma_{1}$, the condition $c$ ) is fulfilled for a range of radii $R_{1} \leq R \leq R^{*}$ where $R_{1}=(4 \sqrt{5} / 3 \pi) v^{1 / 2} N\left[\cos \left(\frac{1}{3} \arctan \sqrt{\left(\frac{\sigma_{2}}{\sigma}\right)^{2}-1}\right)-\right.$ $\left.\sqrt{3} \sin \left(\frac{1}{3} \arctan \sqrt{\left(\frac{\sigma_{2}}{\sigma}\right)^{2}-1}\right)\right]$ and $\sigma_{2}=(8 \sqrt{5} / 27 \pi) v^{1 / 2}$. For $\sigma>\sigma_{1}$, the parabolic solution for the monomer volume fraction is not valid and it has to be set equal to 1 near the surface. However this remains a marginal effect and concerns a very small range of values of the parameters $(\sigma, R)$, since the mean field treatment is valid in the limit of weak $v$ and at moderately high $\sigma$ (formally for $\left.v^{2} \ll \sigma \ll 1\right)$.

36. D. Izzo, C. M. Marques, Macromolecules 30, 6544 (1997).

37. At a scaling level of description, the brush height and the free energy are constant for the whole regime 2 within numerical coefficients of order unity. An attempt at calculating the slight increase of the brush height with decreasing sphere radius can be made by minimizing the Alexander free energy:

$$
\widetilde{F} \cong \Phi^{1 / 4} \frac{L^{2}}{a^{2} N}+N \Phi^{5 / 4}
$$

where $\Phi$ is related to the brush height $L$ through $\Phi=$ $4 \pi R^{2} a N \sigma / V$, where $V=4 / 3 \pi\left[R^{3}-(R-L)^{3}\right]$ is the volume occupied by the brush. At first order, the brush height increment can be written as $L / L_{\text {flat }}-1 \cong \frac{1}{3} a N \sigma^{1 / 3} / R$. This result is incorrect, as can be seen by comparison with equation (25). Indeed, the monomer concentration is assumed to be uniform in this scaling description. Hence, the concentration near the edge of the brush, which contributes slightly more to the present effect of curvature, is overestimated. That is the reason for the overestimated factor $\frac{1}{3}$ instead of $\frac{1}{4}$. The correction to the scaling law $\Phi \cong \sigma^{2 / 3}$ is, at first order

$$
\Phi \cong \sigma^{2 / 3}+\frac{2}{3}\left(\frac{a N \sigma}{R}\right)
$$

Note that this development is valid in the regime 2 that is for $R \gg a N \sigma^{1 / 3} \cong R^{*}$.

38. S. T. Milner, T. A. Witten, J. Phys. France 49, 1951 (1988).

39. We recover the old result of stability of symmetrically polymer decorated membrane under fluctuations of large wavelength (at a mean field level) [38]. However, for surfactant vesicles, the grafting is at thermodynamic equilibrium. Thus, in general, the grafting density is not symmetric 12. This explains why polymer decorated membranes form spontaneously vesicles.

40. J. I. Martin, Z.-G. Wang, J. Phys. Chem. 99, 2833 (1995).

41. P.-G. de Gennes, Scaling Concepts in Polymer Physics, (Cornell Univ. Press, Ithaca, 1979).

42. P.-G. de Gennes, Macromolecules 141637 (1981); Macromolecules, 15, 492 (1982).

43. Since the exponents for the volume fraction $\phi$ in the elastic and in the osmotic terms of the free energy are slightly higher in scaling than in mean-field, the brush tends to avoid presenting any high concentration region : it is rather distributed more evenly. For example, in the parabolic profile presented in reference [34], the scaling profile is slightly 
more flattened in the high $\phi$ region near the wall than the mean-field profile, and as a compensation, it is slightly more concentrated in the low volume fraction region near the edge of the brush.

44. M. Aubouy, Ph. D. Thesis, Université Paris VI, 1995.

45. M. Aubouy, G. H. Fredrickson, P. Pincus, E. Raphaël, Macromolecules 28, 2979 (1995).

46. S. Alexander, J. Phys. Paris 38, 983 (1977).

47. P.-G. de Gennes, Macromolecules 13, 1069 (1980).

48. This is somewhat similar to the collapsed structure of a chain in a poor solvent, which is Gaussian both below and above $\xi$, and confined for number of monomers larger than $G$. In the case of the globule in poor solvent, the expressions for $\xi \simeq a \phi^{-1}$ and $G \simeq(R / a)^{2}$ differ somewhat from the situation studied here.

49. P. Guenoun (private communication).

50. C. Gay, Macromolecules 30, 5939 (1997).

\section{Figure Captions.}

Figure 1: (a) Conformation of a convex brush in the Daoud-Cotton scaling description. The geometry ensures the equilibrium between elastic and osmotic forces. The same picture transposed to concave surfaces (b) is used in references $18,20,24$. The inset shows the monomer volume fraction profile.

Figure 2: Various regimes for a spherical concave grafted polymer brush. The inverse of curvature is $R$ and the grafting density $\sigma$. A schematic representation of the brush is shown using blobs defined in Section 3. This figure is only qualitative. (1: mushrooms, 2 : weak concave brush, 3 : compressed brush, 4: collapsed brush, in gray: unphysical regions)

Figure 3: Variations of the free energy per chain $\widetilde{F}(x)$ for three different values of $R / R^{*}$ for $N=1000, \sigma=0.03$ $\left(R^{*} / a=300.07\right)$ : from bottom to top $R=2 R^{*}$ (region 2 ); $R=R^{*} ; R=0.8 R^{*}$ (region 3 ). The dashed part of the curve corresponds to an unphysical situation [33].

Figure 4: Concentration profile of the brush. (a) Large sphere $\left(R=2 R^{*}\right.$, regime 2$)$ : the profile is parabolic and the concentration vanishes at some finite distance $x^{*}$ from the surface of the sphere (see Eq. (19)). (b) At $R=R^{*}$, the concentration vanishes precisely at the center of the sphere. (c) Small sphere $\left(R=0.8 R^{*}\right.$, regime 3$)$ : truncated parabolic profile with a finite concentration at the center of the sphere (see Eq. (27)).

Figure 5: Variation of the normalized chain end density distribution $\rho$ vs. $r_{0} / R$ for two different radii, $R=$ $1.27 R^{*}$ (regime 2 ) and $R=0.75 R^{*}$ (regime 3$)(N=1000$, $f=600)$.

Figure 6: Variation of the solution $x^{*}$ of equation (15) vs the normalized curvature $R^{*} / R$. The inset shows the variation of the normalized brush height $L / L_{\text {flat }}$ vs the normalized curvature of the sphere $R^{*} / R$.

Figure 7: Variation of the scaled total free energy per chain $\psi$ (see Eq. (19)) in regimes 2 and 3 vs the normalized curvature $R^{*} / R$. Are also represented the elastic $\left(\psi_{e l}\right)$ and osmotic $\left(\psi_{o s}\right)$ parts (where $\psi=\psi_{o s}+\psi_{\text {el }}$ )

Figure 8: Mean square distance between two monomers from the same chain (radial component), as a function of the number of monomers between them (logarithmic scales). (a) In regime 3 , for a given average degree of stretching $|d r / d n|$ and volume fraction $\phi$ (thin line), the thick line is the scaling picture. At long distances, the dependence is linear, which reflects the fact that the chain is stretched (exponent 1). At smaller distances, the chain 
takes the conformation it would have in a semi-dilute solution of volume fraction $\phi$ : it is swollen at small length scales (exponent $3 / 5$ ) and it is Gaussian at length scales larger than $\xi=a \phi^{-3 / 4}$ (exponent $1 / 2$ ). (b) In regime 4, the chains are compressed : the SCF approach does not apply and the scaling approach yields the following picture. For a given volume fraction $\phi$, all chains behave alike. They are swollen at small length scales (exponent 3/5) and Gaussian at length scales larger than $\xi=a \phi^{-3 / 4}$ (exponent $1 / 2$ like in a semi-dilute solution). A $G$-monomer strand spans over the entire interior of the sphere of size $R$ and thus larger strands do not extend any further : they are confined (exponent 0).

Figure 9: Various regimes for a cylindrical concave grafted polymer brush. The inverse of curvature is $R$ and the grafting density $\sigma$. This figure is only qualitative. (1: mushrooms, 1bis: compressed mushrooms, 2: weak concave brush, 3: compressed brush, 4: semi-dilute brush) 
This figure "Figure1revDC.gif" is available in "gif" format from: http://arxiv.org/ps/cond-mat/0102092v2 
This figure "Figure2diagr.gif" is available in "gif" format from: http://arxiv.org/ps/cond-mat/0102092v2 
This figure "Figure3Fx.gif" is available in "gif" format from: http://arxiv.org/ps/cond-mat/0102092v2 
This figure "Figure4parabolic.gif" is available in "gif" format from: http://arxiv.org/ps/cond-mat/0102092v2 
This figure "Figure5densite.gif" is available in "gif" format from: http://arxiv.org/ps/cond-mat/0102092v2 
This figure "Figure6xstar.gif" is available in "gif" format from: http://arxiv.org/ps/cond-mat/0102092v2 
This figure "Figure7energy.gif" is available in "gif" format from: http://arxiv.org/ps/cond-mat/0102092v2 

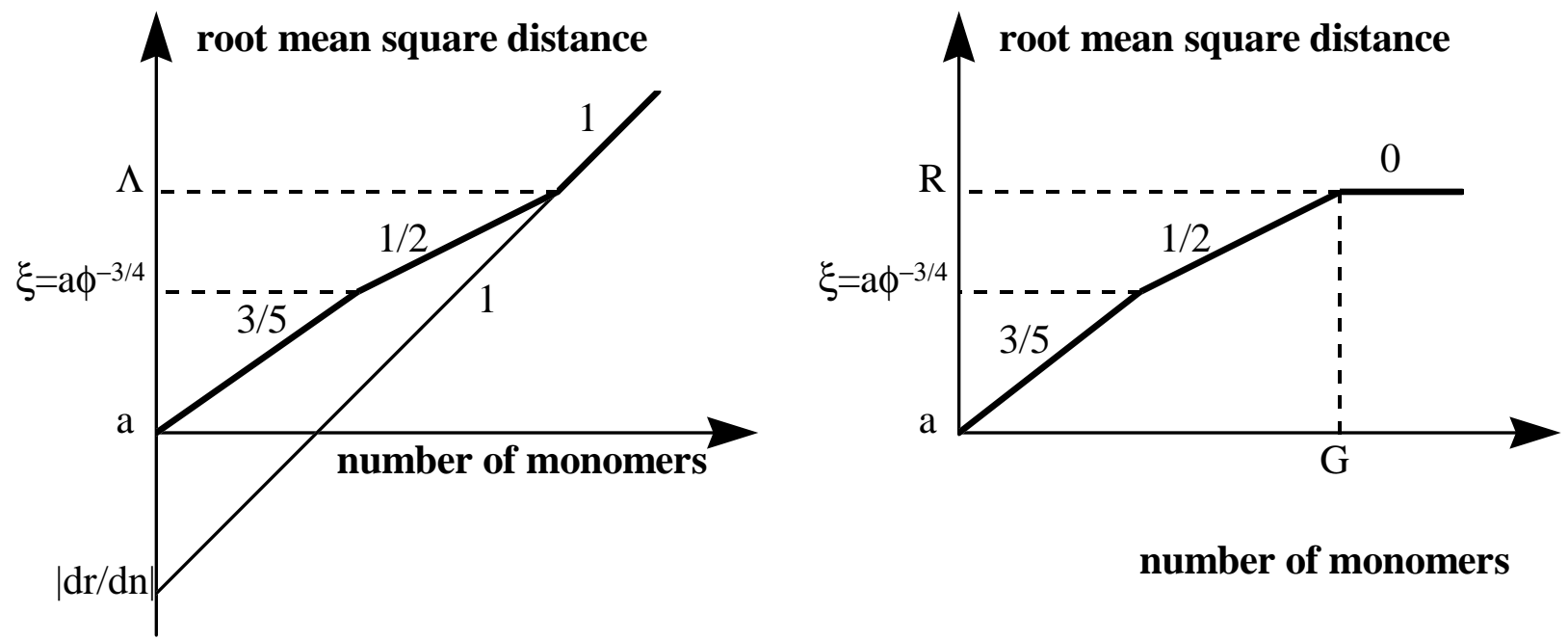
This figure "Figure9cyl.gif" is available in "gif" format from: http://arxiv.org/ps/cond-mat/0102092v2 